\title{
Envelope density pattern around wide binary AGB stars: a dynamical model ${ }^{\star}$ (Research Note)
}

\author{
J. H. $\mathrm{He}^{1,2}$ \\ 1 National Astronomical Observatories/Yunnan Observatory, Chinese Academy of Sciences, PO Box 110, Kunming, \\ Yunnan Province 650011, PR China \\ 2 Academia Sinica, Institute of Astronomy and Astrophysics, PO Box 23-141, Taipei 10617, Taiwan, PR China \\ e-mail: jhhe@asiaa.sinica.edu.tw \\ Received 23 September 2006 / Accepted 10 November 2006
}

ABSTRACT

\begin{abstract}
Context. Various morphologies such as multi-concentric shells and spiral-like patterns have been observed around Proto-Planetary Nebulae and AGB stars. It is widely argued that the regular density patterns are produced by binary systems.

Aims. The goal is to build up a simple dynamical model for the out-flowing circumstellar envelope around AGB stars in a wide binary system to explore the parameter dependence of the geometrical characteristics of column density patterns.

Methods. For an AGB star in a wide binary system, the orbital motion of the star can be approximated as a series of pistons that simultaneously push out dust and gas along radial directions, but work in different oscillation phases. The piston model can fast produce column density patterns with high enough spatial resolutions for parameter dependence exploration.

Results. The formation of 3D quasi-spherical density structure is induced by orbital motion of the AGB star. The column density pattern only depends on two parameters: eccentricity of the orbit $e$ and the terminal outflow velocity to mean orbital velocity ratio $\gamma$. When viewed perpendicular to the orbital plane, spiral, broken spiral, and incomplete concentric shell patterns can be seen, while when viewed along the orbital plane, alternative concentric half-shell, egg-shell, and half-shell half-gap patterns will develop. Nonzero eccentricity causes asymmetry, while larger $\gamma$ makes a weaker pattern and helps bring out asymmetry. A spiral pattern may becomes broken when $e>0.4$. The spiral center is always less than $12 \%$ of spiral pitch away from the orbit center. One should have more chances $(\sim 80 \%)$ seeing spiral-like patterns than seeing concentric shells $(\sim 20 \%)$ in the circumstellar envelope of wide binary AGB stars.
\end{abstract}

Key words. stars: AGB and post-AGB - stars: circumstellar matter - stars: binaries: general - shock waves

\section{Introduction}

In recent years, various kinds of morphology in circumstellar envelopes of AGB stars have been revealed by high spatial resolution observations. For example, Mauron \& Huggins (2000) showed a series of shells in the envelope of the carbon rich AGB star IRC +10216 in an optical image. Balick et al. (2001) showed clear multi-shell structures in the relic AGB envelope around the planetary nebula NGC 6543, the Cat's Eye. Kwok et al. (1998) found multi-arcs near the two lobes of a bipolar proto-planetary nebula IRAS 17150-3224. High velocity bipolar jets had also been discovered in the AGB circumstellar envelope by means of interferometry of maser emission, e.g., the observations by (Imai et al. 2002, 2005) towards W 43A. The most marvelous pattern found most recently is the spiral pattern found by Mauron \& Huggins (2006) in the envelope of AFGL 3068, a C-rich AGB star. They fitted an Archimedes' spiral to the observed spiral pattern, which demonstrated the amazing regularity of the pattern.

The mechanism that produces the various morphologies is still controversial. Steffen \& Schönberner (2000) proposed periodic change of mass-loss rate or wind speed, but the change of mass-loss rate by thermal pulse has too long a period $\left(\sim 10^{5} \mathrm{yr}\right)$. Balick et al. (2001) attributed the multi-shells around the Cat's

* Figure 3 and Appendix are only available in electronic form at http://www . aanda. org
Eye to instability of physical and chemical processes during dust formation and growth, as suggested by Simis et al. (2001), who tried to explain the concentric shells around IRC +10216 in the same way. Mauron \& Huggins (2006) preferred to explain their spiral pattern in AFGL 3068 by numerical results from Mastrodemos \& Morris (1999, MM99 hereafter), in which an AGB star in a wide binary produces a spiral-like density pattern by the reflex motion of the star (their model 4). MM99 performed smooth particle hydrodynamic (SPH) simulations to explore the effects of binarity on AGB wind for circular orbit binaries. In their wide binary example, they showed spiral and concentric shell density patterns formed in the circumstellar envelope. But to understand the observed patterns better, it's helpful to know, if the orbit is not circular, what will be different in the patterns. However, due to difficulties in SPH simulation, e.g., time consuming, not high enough spatial resolution, varying of the particle's smoothing length, etc., a simpler simulation is needed for extensive parameter exploration tasks.

Soker (1994) discussed the influence of a wide binary on the structures of planetary nebulae by taking the AGB mass-loss as a sequence of mass loss pulses. With far distance piston approximation and sticky particle model in my work, the influence of binarity will be explored in more detail. Section 2 gives the numerical piston model to simulate the formation of density pattern in the circumstellar envelope of a wide binary AGB star. Numerical results are present in Sect. 3. A brief summary is given in Sect. 4. 


\section{Piston model and column density pattern}

\subsection{Piston model}

To simplify the problem, several assumptions about an AGB star are made: mass-loss rate does not change with time; outflow velocity is constant and equal to terminal velocity $V_{\mathrm{e}}$; gas pressure can be neglected; and dust gas interaction can be neglected. Although the mass-loss rate of AGB stars may change with time due to thermal pulse, it changes on a very long timescale (e.g., $10^{5}$ years, as suggested by Steffen \& Schönberner 2000) that is much longer than the orbital period. Mass-loss rate and outflow velocity may change due to the interplay of dust formation and dynamics (Simis et al. 2001), but it is not clear whether such a phenomenon really occurs. The assumption of constant outflow velocity is equivalent to assuming that the gravity force and radiation pressure on dust grains and gas are balanced everywhere. This is supported by hydrodynamic calculations (e.g., the model results for IRC +10216 by Skinner et al. 1999), unless one examines the region very close the star. Gas pressure can broaden any density structure by means of thermal dissipation, but its effect is marginal in the outer part of the supersonic outflow. And gas pressure does not alter the geometry of density structures, which is the main topic of this work. Gas and dust are not distinguished and the material is always called gas. These assumptions sound plausible for this work because hydrodynamic effects on the pattern geometry become unimportant in wide binary systems.

Under these assumptions, the motion of each chunk of ejected gas can be treated as ballistic motion. One can imagine that many gas parcels are ejected from the star surface with constant velocity $V_{\mathrm{e}}$ relative to the star. The absolute velocity of each gas parcel in the center of mass $(\mathrm{CoM})$ frame of the binary is equal to the sum of $V_{\mathrm{e}}$ and star motion velocity.

This work only considers density patterns formed far away from the star in the circumstellar envelope. In this case, the star motion and mass loss can be approximated as a piston that is ejecting gas parcels and moving back and forth along radial direction to modulate the velocity distribution in the ejected gas. The ejected gas will be compressed by the velocity gradient to form a density enhancement. Shock may happen when gas parcels collide with each other due to different velocities. One such piston works in one direction. The whole three-dimensional AGB mass-loss envelope can be imagined as a collection of pistons pointing in different directions and running in different phases. The different piston phases in different directions concatenate the density enhancements or shocked regions to form a three-dimensional quasi-spherical density structure, like the structure produced by the SPH simulations by MM99 for circular orbit in their model 4 . This is called a far distance piston approximation.

The orbital motion of the AGB star is assumed to be Keplerian. Projection of the star motion along any given direction $(\theta, \phi)$ (see definition of quantities in Fig. 1) gives the position of the piston along that direction as

$l(t)=a_{\mathrm{p}}\left(\cos E_{\mathrm{p}} \cos \phi+\sqrt{1-e^{2}} \sin E_{\mathrm{p}} \sin \phi\right) \sin \theta$.

Here $e$ and $a_{\mathrm{p}}$ are the eccentricity and semi-major axis of the star orbit, respectively, while $E_{\mathrm{p}}$ is the eccentric anomaly of the star. Then the speed of the piston is

$$
V_{l}(t)=\frac{\mathrm{d} l(t)}{\mathrm{d} t}=\omega a_{\mathrm{p}} \frac{\sqrt{1-e^{2}} \cos E_{\mathrm{p}} \sin \phi-\sin E_{\mathrm{p}} \cos \phi}{1-e \cos E_{\mathrm{p}}} \sin \theta
$$

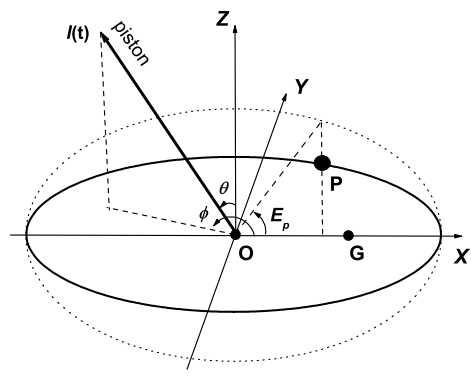

Fig. 1. Schematic illustration of the orbital motion of AGB star (point P), with definitions of Cartesian coordinates $\mathrm{OXYZ}$, eccentric anomaly $E_{\mathrm{p}}$, and position angle $(\theta, \phi)$ of the piston (the heavy arrow). Point $\mathrm{O}$ is the center of the orbit while $\mathrm{G}$ is the center of mass. The dotted circle is the circumscribed circle of the orbit.

where $\omega=2 \pi / T$ is the mean angular velocity of the star motion with orbital period $T$. The time dependence of piston motion is reflected by

$E_{\mathrm{p}}-e \sin E_{\mathrm{p}}=\omega t$.

In the numerical simulation, the $4 \pi$ solid angle is divided into 10000 equally sized patches and a piston is computed along the center direction of each solid angle element. Each piston is assumed to eject 100 gas parcels (with equal mass) within one orbital period. Gas parcels ejected at different times set out from different positions $l(t)$ with different velocities $V_{\mathrm{e}}+V_{l}(t)$. After leaving the star, each gas parcel moves outward with constant speed, until it collides with neighboring parcels. The collision of gas parcels causes shock and the collided gas parcels are arbitrarily assumed to merge with each other (actually move together side by side with very small fixed distances in the simulation, sticky particles). The velocity of the merged gas parcels is determined by conservation of momentum. The spatial distribution of all gas parcels represents the 3D density distribution of outflowing material in the AGB envelope. Projection of the 3D distribution of gas parcels onto any specified sky plane produces a column density pattern.

\subsection{Normalization of the problem}

Several physical parameters are involved in the piston model: orbital parameters such as semi-major axis $a_{\mathrm{p}}$, eccentricity $e$, and period $T$, and the steady outflow velocity $V_{\mathrm{e}}$. However, not all of them are independent of the problem. To reduce the number of parameters, the above formulae need be normalized by dividing all length quantities by the circumference of the circumscribed circle of the orbit $2 \pi a_{\mathrm{p}}$, dividing all time quantities by period $T$, and dividing all velocity quantities by the mean orbital velocity of the star $\omega a_{\mathrm{p}}$. Then, the piston motion formulae Eqs. (1) and (2) become

$$
\begin{aligned}
& \tilde{l}(\tilde{t})=\frac{1}{2 \pi}\left(\cos E_{\mathrm{p}} \cos \phi+\sqrt{1-e^{2}} \sin E_{\mathrm{p}} \sin \phi\right) \sin \theta \\
& \tilde{V}_{l}(\tilde{t})=\frac{\sqrt{1-e^{2}} \cos E_{\mathrm{p}} \sin \phi-\sin E_{\mathrm{p}} \cos \phi}{1-e \cos E_{\mathrm{p}}} \sin \theta
\end{aligned}
$$

and

$E_{\mathrm{p}}-e \sin E_{\mathrm{p}}=2 \pi \tilde{t}$.

In the numerical simulation, each gas parcel is ejected at normalized position $\tilde{l}(\tilde{t})$ with the constant normalized speed $\gamma+\tilde{V}_{l}(\tilde{t})$ (with $\gamma=V_{\mathrm{e}} / \omega a_{\mathrm{p}}$ being a velocity ratio). After the normalization, one has only two free model parameters: $e$ and $\gamma$. All other parameters such as $a_{\mathrm{p}}, T$, and $V_{\mathrm{e}}$ are combined in the parameter $\gamma$. 

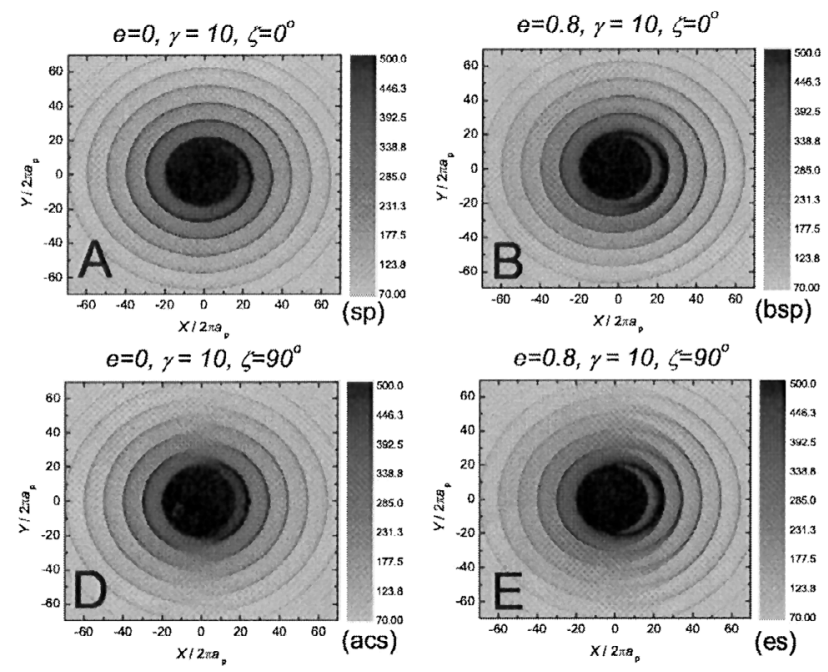

\subsection{Formulation of single piston density pattern}

Thanks to the simplicity of the piston model, it is possible to directly formulate the density pattern geometry (position of density peaks or shocked regions) in the outflowing gas. In some cases, solving the formulas may be easier than analyzing numerical column density maps in the discussion of certain aspects of the pattern geometry. But due to the limitation of space, the deduction of normalized pattern formulas is put in Appendix A that is available only in electronic version.

\section{Results}

\subsection{Numerical column density patterns}

Column density pattern geometry has been explored in a large range of parameter space: $e=0.0-0.8, \gamma=10-90$, and different observers' direction angles $(\zeta, \psi)$. Representative types of morphology found in the computed column density maps are shown in Fig. 2. In the first row patterns are viewed perpendicular to the orbital plane, while in the second row patterns are viewed along the orbital plane. Maps A and D show that, for the circular orbit case, the column density pattern is a good spiral-like pattern when viewed perpendicular to the orbital plane and alternative concentric half shell pattern when viewed along the orbital plane, which closely resembles the SPH simulation results of MM99 (their Model 4). Comparing map A with B tells us that, when eccentricity is non-zero, the spiral pattern may become broken in its inner part (the broken spiral pattern in map B). The extent of the spiral break decreases when the shock pattern moves outwards, and eventually continuous spiral forms appear in the outer part of the pattern. If both $e$ and $\gamma$ are large, as the case of map $\mathrm{C}$, even if observed perpendicular to the orbit plane, the column density pattern still looks like a series of concentric shells, but the shells are not only much fainter, but also incomplete in the lower-right quarter (incomplete concentric shell pattern).

A common character of the patterns in the second row of Fig. 2 is concentric shells with the lack of density pattern along the vertical directions, which are the directions of the orbit pole. Comparing maps D and E tells us that non-zero orbital eccentricity may cause the variation of relative radii of the concentric halfshells and make strong non-shocked patterns around shocked
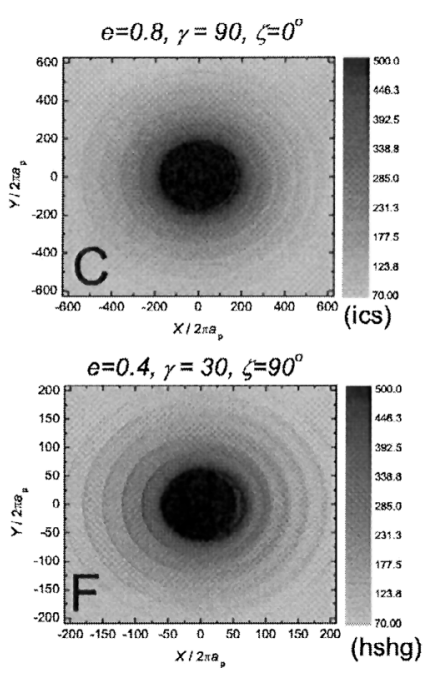

Fig. 2. Column density patterns calculated by the piston model for representative cases. Observer's azimuth angle is always $\theta=-90^{\circ}$, which means the pericenter of star orbit is pointed to the right. Inclination angle $\zeta$ and $e, \gamma$ are shown on top of each map. All maps are in linear stretch in the same gray scale range. $X$ and $Y$ axes are in normalized length unit (i.e., times of $\left.2 \pi a_{\mathrm{p}}\right)$. Pattern types shown at the right bottom of each map are: $s p$ - spiral; $b s p$ - broken spiral; ics - incomplete circular shells; acs - alternative concentric shells; es - egg shells; and hshg - half-shell half-gap.

patterns. As a result, in map $\mathrm{E}$, the radii of the left and right series of half-shells are nearly the same, which imitate nested egg shells. In the right half of map E, the innermost shells look thicker. They are actually double shells with non-shocked gas filled in between. Moving outwards, the double shells eventually merge with each other to form sharp single-layer shocked shells. One should also have noticed the left-right asymmetry both in density contrast and in pattern shape in map E, which is the effect of non-zero eccentricity, too. Comparing map F with maps $\mathrm{D}$ and $\mathrm{E}$ gives us a surprise: the larger $e$ and $\gamma$ in map $\mathrm{F}$ cause the missing of shells on the right side, which results in a half-shell half-gap pattern.

Exploration of more maps shows that left-right asymmetry (as in the broken spiral, incomplete concentric shell, egg shell, half-shell half-gap patterns) only appears when $e>0$ and $\gamma$ is large, otherwise, when $\gamma$ is small, even if $e>0$ and the asymmetry exists, the asymmetry may be buried in the invisible central region of the maps. When the asymmetry is prominent, its dependence on the observer's azimuth angle is also found when observing along the orbital plane: the pattern looks more left-right symmetrical when being viewed along the major orbit axis, while it appears more left-right asymmetrical when being viewed along the minor orbit axis. Several maps that illustrate this effect are present in Fig. 3 in the electronic version only.

\subsection{Several interesting properties of the column density patterns}

One of the interesting problems is how the transition from spirallike pattern to concentric half shell pattern happens when the observer's inclination angle changes from $0^{\circ}$ to $90^{\circ}$. In Fig. 4 , more column density patterns are shown for different observation inclination angles $\zeta=0^{\circ}, 68^{\circ}, 79^{\circ}$, and $90^{\circ}$ for the circular orbit case. One can see that only when the inclination angle is $\zeta \geq 79^{\circ}$, does the column density pattern begin to prominently deviate from the spiral-like pattern. That is to say, in a large range of inclination angles, from $0^{\circ}$ to $79^{\circ}$, one can see a spiral-like pattern. If one sees a concentric shell pattern, the inclination angle of observation must be within the $90 \pm 10$ degree range, that is, the star orbit must be almost edge on. If the distribution of the star orbit orientation is completely random, one has an approximate probability of $1-\cos 79^{\circ}=81 \%$ seeing the spiral-like pattern 

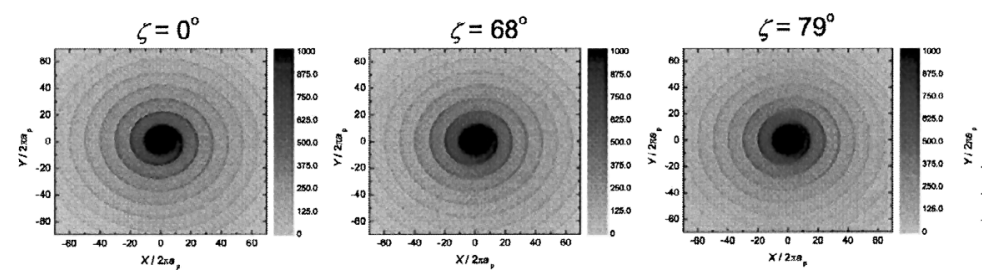

Fig. 4. Maps show inclination angle effects. Azimuth angle is $\psi=-90^{\circ}, e=0$, and $\gamma=$ 10 for all maps. One can see the spiral pattern in the inclination angle range of $0^{\circ}-79^{\circ}$ and the concentric shells in the $79^{\circ}-90^{\circ}$ range.

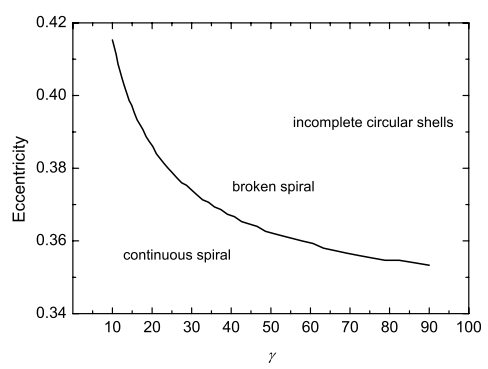

Fig. 5. The border line divides the $\gamma-e$ parameter space into two parts: the density pattern in the orbital plane of the model is a continuous spiral pattern in the lowerleft part and a broken spiral or incomplete concentric shell pattern in the upperright part. and only a probability of $19 \%$ seeing the concentric shell pattern in the circumstellar envelope of such a wide binary AGB star. MM99 intuitively speculated that, when viewed with finite resolution at great distances from the star and from some intermediate latitude, the multi-shells may appear to form full circles. This is contradicted with the conclusion of this work and is incorrect. Actually the relative shift of shells with inclination angle is true but the shift of shells forms a spiral pattern, instead of full rings. This can be easily understood because one sees spirals when observing perpendicular to the orbit plane and because the left and right shells have different radii.

Another question is when the spiral pattern becomes a broken spiral. The answer lies in the single piston model along the pericenter direction of the orbit because the broken part of a spiral always happens on the pericenter side. Some trial solutions of single piston formulas (A.4, A.5, A.6 in Appendix A) demonstrate that the formation of the broken spirals is due to a double solution of $E_{\mathrm{p}}$ within one period. These formulas are numerically solved for all possible combinations of $(e, \gamma)$. Those pairs of $(e, \gamma)$ beyond which a double solution of $E_{\mathrm{p}}$ begins to appear are shown in Fig. 5 as a border line that divides the parameter space into two halves: lower left half for the continuous spiral; upper right half for the broken spiral and the incomplete concentric shell pattern. Because $e$ does not change too much around 0.4 along the border line, one may say that a broken spiral pattern may appear when eccentricity $e \geq 0.4$.

The third question is where the spiral center is. Archimedes' spiral curve is fitted to the mature part of shocked patterns computed from the piston model in a wide range of parameter space $(e=0-0.8, \gamma=2-90)$. The pattern fitting demonstrates that the shocked patterns are always good Archimedes' spiral curves. The center of the spiral is not at the orbit center, but the shift of the spiral center from the orbit center shows some degree of dependence on both parameters $e$ and $\gamma$. Generally speaking, higher eccentricity and smaller $\gamma$ causes larger spiral center shifts. However, in most cases, the spiral center shift is always less than $2 \%$ of a spiral pitch in the major orbit axis direction and always less than $12 \%$ of a spiral pitch in the minor orbit axis direction, which means the spiral center should be just on the star position when the observation resolution is not extremely high. In circular orbit cases, the spiral center is exactly at the orbit center.
The properties of the various column density patterns may be critical in distinguishing the binary mechanism from the massloss rate modulation mechanism. The patterns also serve as a magnifier to show the binary motion in the center of the circumstellar envelope. On the other hand, the formation of the density pattern, especially the shocked density pattern, greatly enhances the visibility of the circumstellar material and enables us to study the mass-loss history of AGB stars in binary systems.

\section{Summary}

A piston model is used to simulate the quasi-spherical density patterns formed in the supersonic outflow of wide binary AGB stars. The simple and quick model allows parameter space exploration in detail. Normalization of the piston model shows that the normalized 3D density pattern in the circumstellar envelope only depends on two parameters: eccentricity of the star orbit $e$ and steady outflow velocity to mean star motion velocity ratio $\gamma\left(=V_{\mathrm{e}} / \omega a_{\mathrm{p}}\right)$. When viewed perpendicular to the orbital plane, the spiral-like pattern, broken spiral pattern, and incomplete concentric shell pattern can be seen, while when viewed along the orbital plane, the alternative concentric half-shell pattern, egg-shell pattern, and half-shell half-gap pattern can be seen. Higher eccentricity causes more left-right asymmetry and the variation of relative radii between the left and right series of concentric half shells, while larger $\gamma$ makes a weaker density pattern but helps bring out asymmetry from the pattern center.

One sees a spiral-like pattern in a large observers' inclination angle range $\left(0^{\circ}-79^{\circ}\right)$, while concentric half-shell patterns can be seen only in edge-on binary, which indicates that one has more of a chance $(\sim 81 \%)$ seeing spiral-like patterns in the sky than seeing concentric shells ( $\sim 19 \%$ chance). Fitting Archimedes'spiral curve to numerical shocked column density patterns demonstrates that the spiral center is away from the orbit center by no more than $12 \%$ of the spiral pitch. The spiral pattern may become broken when the eccentricity is larger than about 0.4 .

Acknowledgements. I thank the anonymous referee who helped me to condense and improve the descriptions in this work. This work is partly supported by the National Natural Science Foundation of China under Grant Nos. 10433030 and 10503011 .

\section{References}

Balick, B., Wilson, J., \& Hajian, A. R. 2001, AJ, 121, 354

Imai, H., Nakashima, J.-I., Diamond, P. J., Miyazaki, A., \& Deguchi, S. 2005, ApJ, 622, L125

Imai, H., Obara, K., Diamond, P. J., Omodaka, T., \& Sasao, T. 2002, Nature, 417, 829

Kwok, S., Su, K. Y. L., \& Hrivnak, B. J. 1998, ApJ, 501, L117

Mastrodemos, N., \& Morris, M. 1999, ApJ, 523, 357

Mauron, N., \& Huggins, P. J. 2000, A\&A, 359, 707

Mauron, N., \& Huggins, P. J. 2006, A\&A, 452, 257

Simis, Y. J. W., Icke, V., \& Dominik, C. 2001, A\&A, 371, 205

Skinner, C. J., Justtanont, K., Tielens, A. G. G. M., et al. 1999, MNRAS, 302, 293

Soker, N. 1994, MNRAS, 270, 774

Steffen, M., \& Schönberner, D. 2000, A\&A, 357, 180 


\section{Online Material}



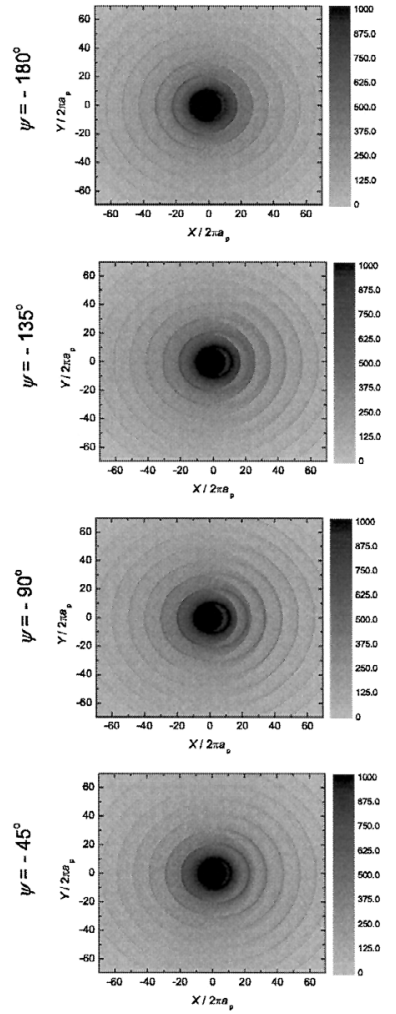

Fig. 3. Computed maps show the azimuth angle effects on pattern geometry. All maps are shown for $e=0.8, \gamma=10, \zeta=-90^{\circ}$, that is, viewed along the orbit plane (edge on). $\psi=-180^{\circ}$ means viewing along the major orbit axis, while $\psi=-90^{\circ}$ means viewing along the minor orbit axis.

\section{Appendix A: Formulation of single-piston density-pattern}

This section only considers the normalized piston model in which the normalized orbital period is 1 and the normalized steady outflow velocity is $\gamma$. Pattern geometry is determined by the positions of all density peaks and/or shock regions. Because the piston motion is periodic, one only needs to consider gas parcels ejected within a single period. Before the occurrence of shock, there should be no collision of gas parcels. One may find a peak parcel that corresponds to a density maximum. Assuming the peak parcel was ejected at an earlier time $\tilde{t}_{\mathrm{p}}$ (the peak parcel ejection time), the radial position of the peak parcel (or the density peak) at a given time $\tilde{t}$ is

$\tilde{r}_{\text {peak }}(\tilde{t})=\tilde{l}\left(\tilde{t}_{\mathrm{p}}\right)+\left[\gamma+\tilde{V}_{l}\left(\tilde{t}_{\mathrm{p}}\right)\right]\left(\tilde{t}-\tilde{t}_{\mathrm{p}}\right)$.

Here the gas parcel velocity is expressed as two components: steady outflow velocity $\gamma$ and an individual velocity component $\tilde{V}_{l}\left(\tilde{t}_{\mathrm{p}}\right)$ contributed by the star motion. At a later time $\tilde{t}_{\mathrm{c}}$ when the first collision between gas parcels occurs (the peak parcel is usually the parcel that collides with others first), shock region begins to form. The time $\tilde{t}_{\mathrm{c}}$ is called the shock time, and the parcels that collide first are called the "shock parcels". After that, further gas parcel collisions modify the velocity of the shock parcels according to the conservation of momentum. At any given time $\tilde{t}>\tilde{t}_{\mathrm{c}}$, assuming the shock parcels were ejected at an earlier time $\tilde{t}_{\mathrm{s}}$ (the shock parcel ejection time), the radial position of shock parcels (or shock region) is

$\tilde{r}_{\text {shock }}(\tilde{t})=\tilde{l}\left(\tilde{t}_{\mathrm{s}}\right)+\gamma\left(\tilde{t}-\tilde{t}_{\mathrm{s}}\right)+\tilde{V}_{l}\left(\tilde{t}_{\mathrm{s}}\right)\left(\tilde{t}_{\mathrm{c}}-\tilde{t}_{\mathrm{s}}\right)+\int_{\tilde{t}_{\mathrm{c}}}^{\tilde{t}} \tilde{V}_{\mathrm{m}}(\tilde{t}) \mathrm{d} \tilde{t}$.

The individual velocity component of the shock parcels $\tilde{V}_{\mathrm{m}}(\tilde{t})$ in the last term on the right side is different from star motion velocity $\tilde{V}_{l}\left(\tilde{t}_{\mathrm{s}}\right)$ because collisions modify it according to the convervation of momentum. $\tilde{V}_{\mathrm{m}}(\tilde{t})$ cannot be analytically expressed, but has to be determined by piston model simulation.

To calculate the radial positions of density peaks or shock regions using the above formulas, one needs to know $\tilde{t}_{\mathrm{p}}, \tilde{t}_{\mathrm{s}}, \tilde{t}_{\mathrm{c}}$. They can be determined using the definition of density maximum. Under the assumption of constant mass-loss rate $\dot{M}$ and outflow velocity $\gamma$, the overall density distribution $\tilde{\rho}\left(\tilde{t}, \tilde{t}_{\mathrm{p}}\right)$ in the circumstellar envelope at a given time $\tilde{t}<\tilde{t}_{\mathrm{c}}$ roughly follows an inverse square law of radius $\tilde{r}\left(\tilde{t}_{\mathrm{p}}\right)$ upon which the density patterns are superimposed. Therefore, it is helpful to define a pattern density by multiplying the square of radius by density. It is not difficult to deduce the formula of pattern density as

$\tilde{\rho}^{\prime}\left(\tilde{t}, \tilde{t}_{\mathrm{p}}\right)=\tilde{\rho}\left(\tilde{t}, \tilde{t}_{\mathrm{p}}\right) \tilde{r}^{2}\left(\tilde{t}_{\mathrm{p}}\right)=\frac{\dot{M}}{4 \pi} /\left[\gamma-\frac{\mathrm{d} \tilde{V}_{l}\left(\tilde{t}_{\mathrm{p}}\right)}{\mathrm{d} \tilde{t}_{\mathrm{p}}}\left(\tilde{t}-\tilde{t}_{\mathrm{p}}\right)\right]$.

By constraining the maximum of the pattern density $\tilde{\rho}^{\prime}\left(\tilde{t}, \tilde{t}_{\mathrm{p}}\right)$, one may derive the following formulas for solving $\tilde{t}_{\mathrm{p}}$ :

$$
\begin{aligned}
& \frac{\mathrm{d}^{2} \tilde{V}_{l}\left(\tilde{t}_{\mathrm{p}}\right)}{\mathrm{d} \tilde{t}_{\mathrm{p}}^{2}}\left(\tilde{t}-\tilde{t}_{\mathrm{p}}\right)=\frac{\mathrm{d} \tilde{V}_{l}\left(\tilde{t}_{\mathrm{p}}\right)}{\mathrm{d} \tilde{t}_{\mathrm{p}}} \\
& \frac{\mathrm{d}^{3} \tilde{V}_{l}\left(\tilde{t}_{\mathrm{p}}\right)}{\mathrm{d} \tilde{t}_{\mathrm{p}}^{3}}\left(\tilde{t}-\tilde{t}_{\mathrm{p}}\right)<2 \frac{\mathrm{d}^{2} \tilde{V}_{l}\left(\tilde{t}_{\mathrm{p}}\right)}{\mathrm{d} \tilde{t}_{\mathrm{p}}^{2}} \\
& 0 \leq \tilde{t}-\tilde{t}_{\mathrm{p}} \leq \gamma / \frac{\mathrm{d} \tilde{V}_{l}\left(\tilde{t}_{\mathrm{p}}\right)}{\mathrm{d} \tilde{t}_{\mathrm{p}}} .
\end{aligned}
$$

When the second inequality in formula (A.6) becomes equality, one can determine the moment when the shock just begins to happen, and therefore the solution of the shock parcel ejection time $\tilde{t}_{\mathrm{s}}=\tilde{t}_{\mathrm{p}}$ can be derived by solving formulas (A.4-A.6) for this moment. In this case, the shock time can also be derived from the second equality of formula (A.6) as

$\tilde{t}_{\mathrm{c}}=\tilde{t}_{\mathrm{s}}+\gamma / \frac{\mathrm{d} \tilde{V}_{l}\left(\tilde{t}_{\mathrm{s}}\right)}{\mathrm{d} \tilde{t}_{\mathrm{s}}}$

With $\tilde{t}_{\mathrm{p}}, \tilde{t}_{\mathrm{s}}, \tilde{t}_{\mathrm{c}}$ solved from the formulas, one can determine the density peak or shock region postions from Eqs. (A.1) and (A.2), and hence the pattern geometry.

Semi-analytical solutions for the special case of circular orbit have been derived on the basis of the formulas. (The solutions are not shown in this paper.) Archimedes' spiral pattern and alternative concentric shell pattern have been confirmed by the analytical solutions only in the outer part of the pattern, while, in the central region of the patterns, deviation from these standard curves is expected. 